\title{
Ultrasonic Assessment of Stabilized Soils
}

\author{
Nazli Yesiller ${ }^{1}$, James L. Hanson ${ }^{2}$, and Mumtaz A. Usmen ${ }^{3}$
}

\section{Abstract}

The feasibility of using ultrasonic testing, in particular P-wave velocities, to evaluate stabilized soils was investigated. A high-plasticity clay soil that was stabilized with lime, cement, and lime - fly ash mixtures was used in the study. The testing program consisted of determination of $P$-wave velocities and compression characteristics of the stabilized soils immediately after compaction and subsequent to 7 days and 28 days of curing. Variation of velocity with stabilizing agent, curing time, and also with the compression characteristics of the soils was investigated. It was observed that $\mathrm{P}$-wave velocities were higher for samples stabilized with cement compared with samples stabilized with lime and fly ash. In addition, velocities increased with curing time for all the stabilized mixes. In general, the velocities of the samples increased as the unconfined compressive strength of the samples increased. However, there was a substantial amount of scatter in the data. The trends observed in the modulus data were better and the P-wave velocities of the stabilized soils increased as the modulus of the soils increased. Also the variation of modulus with time was similar to the variation of velocity with time. In addition, the $\mathrm{P}$-wave velocities increased as the densities of the samples increased.

\section{Introduction}

Chemical soil stabilization is used in various civil engineering applications to improve engineering properties and behavior of soils. The most common stabilizing agents are cement and lime, with fly ash also used as an agent. In most of the applications, improvement in the compression characteristics of soils is required.

\footnotetext{
1 Associate Member, ASCE, Assistant Professor, Department Of Civil and Environmental Engineering, Wayne State University, Detroit, MI 48202

${ }^{2}$ Member, ASCE, Assistant Professor, Department Of Civil Engineering, Lawrence Technological University, Southfield, MI 48075

${ }^{3}$ Member, ASCE, Professor, Department Of Civil and Environmental Engineering, Wayne State University, Detroit, MI 48202
} 
Soil stabilization involves determination of the type and amount of stabilizing agent required and verifying the quality of the resulting stabilized soil. Criteria such as previous experience, regulatory requirements, availability of materials, etc. are used to determine the type and amount of the stabilizing agent to be used for a particular application. In addition, a mixture design procedure is used for the selection of the stabilizing agent. The mixture design procedure consists of preparation of various mixtures of the soil with varying amounts and/or types of stabilizing agents and testing the mixtures. Tests are conducted on these mixtures to determine the compaction characteristics of the soils and also to determine the specific property of the soil to be improved, such as compressive strength. The quality of stabilized soils in the field is verified by procedures that are similar to those used for compacted soils. In most cases, this involves determining the water content and dry density of the soils. In addition, tests can be conducted to determine the specific property of the soil to be improved such as the compressive strength. Extensive literature is available on soil stabilization including materials, testing, and design procedures (Brown 1996).

Mixture design and quality control procedures require extensive testing of the soils. Properties and behavior of chemically stabilized soils are time-dependent. Testing at various times subsequent to mixing the soil with the chemical agent is needed to characterize the soils thoroughly. Commonly, destructive test methods are used to determine properties and the behavior of the soils (ASTM 1992, AASHTO 1997). Nondestructive test methods have not been used much to date to analyze stabilized soils.

Nondestructive test methods can provide a fast and simple alternative approach to analyzing stabilized soils. This study was conducted to assess the feasibility of using ultrasonic testing to evaluate stabilized soils. Ultrasonic velocity was measured on laboratory prepared stabilized soil samples. Variation of ultrasonic velocity with type of stabilizing agent and curing time was investigated. Variation of the velocity with compression characteristics of the stabilized soils was also studied.

\section{Ultrasonic Testing}

Ultrasonic testing was selected as the nondestructive test procedure for this study since it is simple and fast and there is significant experience in the use of this method for evaluating concrete for structural applications.

\section{General Principles}

Ultrasonic testing is used for nondestructive evaluation of materials and structures. Ultrasonic waves are stress waves with frequencies higher than $20 \mathrm{kHz}$ that propagate in mass media. Stress waves are generally introduced into a material using electronic means from an outer surface. Reflected and refracted waves are generated in the material when the incident wave encounters a boundary. These waves are also received from an outer surface. Piezoelectric transducers are used to transmit and receive the stress waves. Typical transducer arrangements include 
pulse-echo test arrangement and through-transmission test arrangement. In pulseecho arrangement, one transducer is used to send and receive the waves. In throughtransmission test method, two transducers are used. Waves are introduced into a material using a transmitting transducer placed on one surface of the material. The waves that propagate through the material are received by the second transducer placed on the opposite surface of the material. This arrangement is typically used for highly attenuating materials such as soils and concrete.

Longitudinal and transverse waves can be transmitted in solid materials. Longitudinal waves are commonly referred to as primary waves or P-waves. When these waves are introduced into a material, particles move in the same direction as the direction of wave propagation. Transverse waves are commonly referred to as shear waves or S-waves. When these waves are introduced into a material, particles move in the direction(s), which is perpendicular to the direction of wave propagation. Elastic constants of materials such as Young's modulus, shear modulus, and bulk modulus can be determined using velocity of propagation of $\mathrm{P}$ and S-waves. In addition, Poisson's ratio can be calculated using these velocities. These properties can be determined for soils using equations developed for elastic materials since the strains generated in soils during ultrasonic testing are very small $\left(<10^{-4} \%\right)$ and the material approximates elastic behavior in this strain range.

Propagation of ultrasonic stress waves in a material is affected by the properties and condition of the material. Transmission of waves in a material is quantified generally using two parameters: velocity and attenuation. Ultrasonic velocity can be correlated to elastic constants and mechanical properties of a material, whereas ultrasonic attenuation can be correlated to microstructural properties of the material (McIntire 1991).

\section{Test Procedure Used in This Study}

The ultrasonic tests conducted for this study consisted of determination of velocity of P-waves in the stabilized soils. The tests were conducted using a measurement system with three units: (1) P-wave transducers, (2) pulser-receiver, and (3) data acquisition system (Figure 1). The 50-kHz-center frequency transducers were connected to a pulser-receiver and a computer for digitization of data. The 10 $\mathrm{MHz}$-bandwidth broadband pulser-receiver had an adjustable repetition rate in the range of $20 \mathrm{~Hz}$ to $2 \mathrm{kHz}$, with a $1 \mathrm{~V}$ output for synchronous triggering of the signal acquisition board during data acquisition. The data acquisition system included a computer equipped with an $A / D$ (analog to digital converter) board with $100 \mathrm{MHz}$ sampling rate and a digital oscilloscope software package which was used for viewing waveforms and for adjusting data acquisition parameters.

The tests were conducted using the through-transmission test method (Figure 1). The waves were sent by a transmitting transducer placed on one end of the sample. The transmitted waves that travel through the entire thickness of the samples were recorded by the receiving transducer placed on the opposite end of the sample. The velocity of the P-waves was obtained as the ratio of the travel path (the 
thickness of the samples) to the travel time of the waves. The thickness of the samples was determined using mechanical measurements (i.e. using a pair of calipers with a resolution of $0.02 \mathrm{~mm}$ ). The travel time was obtained from ultrasonic measurements as the first arrival time of the waves at the receiving transducer. The arrival time was determined with a resolution of $0.01 \mu \mathrm{sec}$.

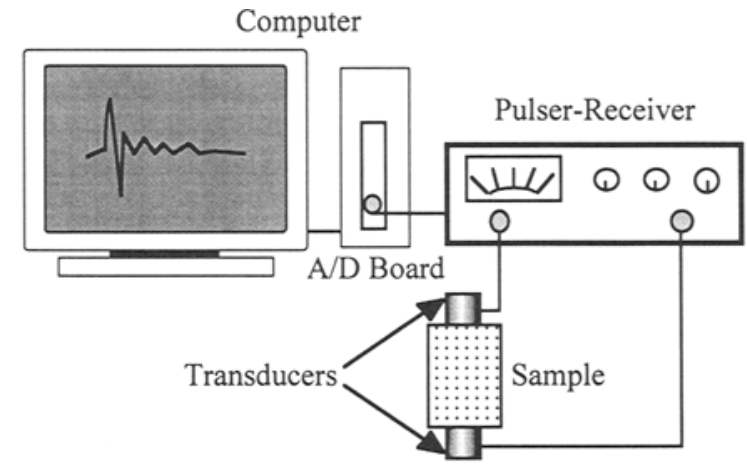

(a) Schematic Representation of Test Setup

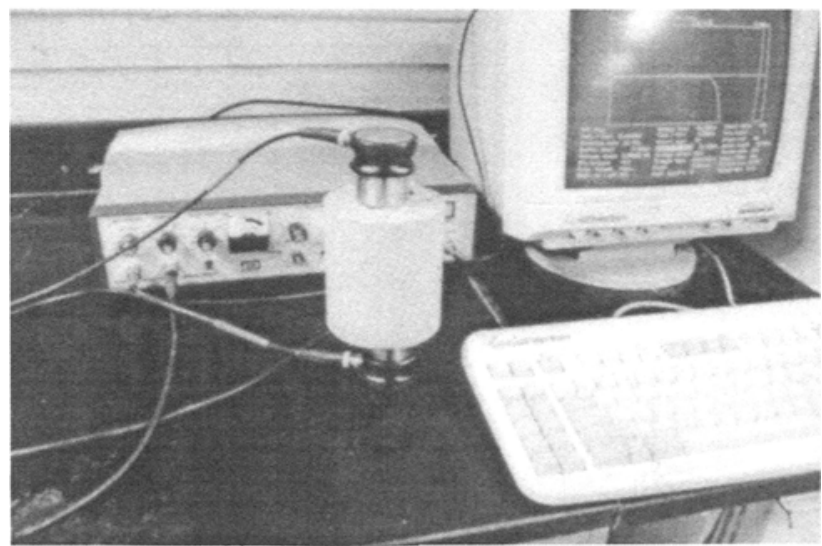

(b) Photograph of Test Setup

Figure 1. Test Setup

The first arrival time was calculated as the difference between the time of application of the pulse by the transmitting transducer and the arrival time of the signal in the receiving transducer. The A/D board was synchronized with the pulser- 
receiver such that data collection started at the time of pulse application. Therefore, there was no uncertainty in the determination of the pulse application time, which was equal to $0 \mu \mathrm{sec}$. Wave averaging and calibration procedures were used to minimize random and systematic errors associated with determination of the first arrival time of the waves. A single waveform recorded in the computer consisted of an average of 32 waveforms. For each measurement, four such waveforms were recorded. The average of these four waveforms was further processed using a computer code to determine the first arrival time. Details of the determination of first arrival time are presented by Inci (2000). This setup was also used effectively to test compacted clayey soils and concrete (Yesiller et al. 2000, Udegbunam 2000). A typical waveform obtained in the tests is presented in Figure 2. The arrival time determined using the computerized procedure is indicated on the waveform.
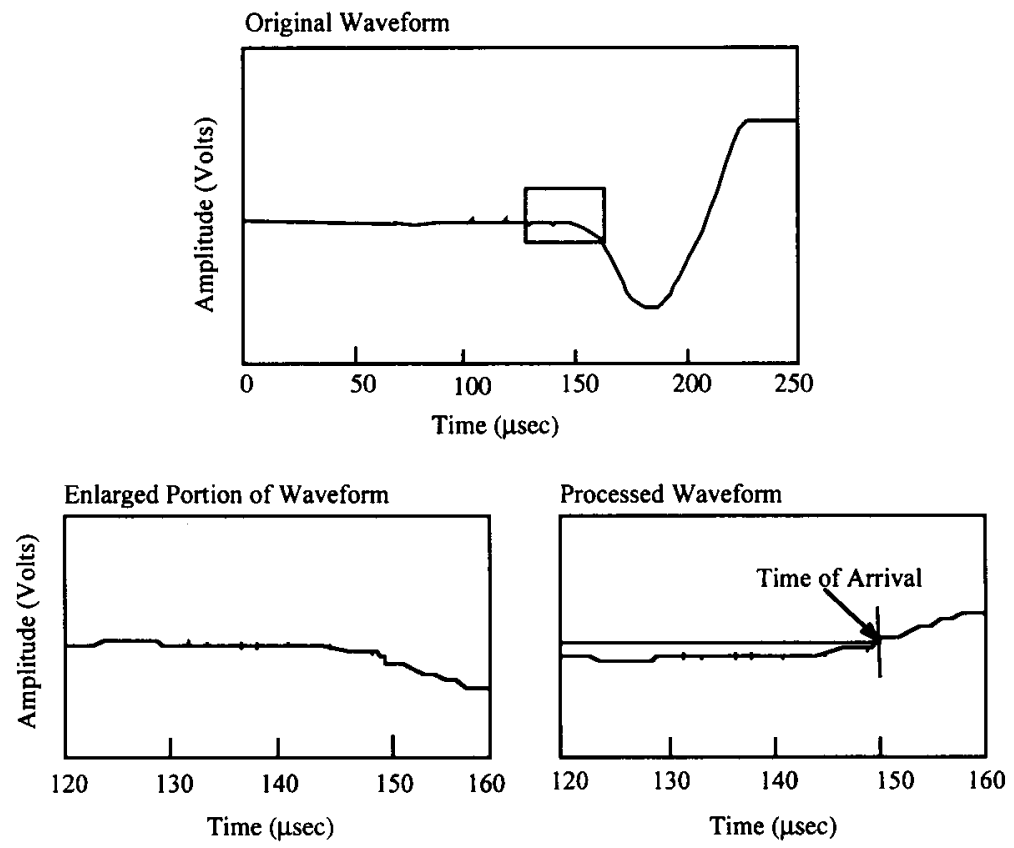

Figure 2. Typical Waveform

\section{Testing Program}

The testing program consisted of the determination of compression characteristics and ultrasonic velocity of a soil stabilized with lime, cement, and lime-fly ash mixtures. The tests were conducted on samples immediately after compaction and also subsequent to 7 days and 28 days of moist curing. 


\section{$\underline{\text { Materials }}$}

Tests were conducted on a high-plasticity clay soil. The soil was manufactured by mixing $40 \%$ sand, $45 \%$ kaolinite, and $15 \%$ bentonite. The engineering properties and compaction characteristics of the soil are presented in Table 1. High-calcium hydrated lime, Type I Portland cement, and Type F fly ash were used as the stabilizing agents. The soil was stabilized with $3 \%$ and $6 \%$ lime; $4 \%$ and $8 \%$ cement; and $4 \%$ lime - $8 \%$ fly ash and $8 \%$ lime - $15 \%$ fly ash mixtures. Compaction characteristics of the stabilized soil mixtures were also determined. It was observed that the optimum water content for the stabilized soils varied between $16 \%$ to $19 \%$ with an average of $17 \%$. Therefore, all of the mixtures were prepared at $17 \%$ water content which is representative of near optimum water content for all of the stabilized mixtures.

Table 1. Soil Properties

\begin{tabular}{c|c|c}
\hline Classification & USCS & CH \\
\hline Particle Size & Sand & 40 \\
\cline { 2 - 3 }$(\%)$ & Clay & 60 \\
\hline \multirow{2}{*}{ Atterberg Limits } & Liquid Limit & 74 \\
\cline { 2 - 3 } & Plasticity Index & 55 \\
\hline Compaction & $\gamma_{\text {dmax }}\left(\mathrm{kN} / \mathrm{m}^{3}\right)$ & 17.7 \\
\cline { 2 - 3 } Characteristics & $\mathrm{w}_{\text {opt }}(\%)$ & 16.5 \\
\hline
\end{tabular}

Tests

The tests consisted of determination of compression characteristics and Pwave velocity of the soils in the laboratory. The tests were conducted on the day of sample preparation and subsequent to 7 days and 28 days of curing.

Water was sprayed on dry soils and mixed to obtain $17 \%$ water content. The mixtures were allowed to hydrate for 24 hours. The stabilizing agents were added to the hydrated soils immediately before compaction. The mixtures were compacted in standard Proctor molds using a mechanical compactor. A total of 72 samples was prepared; 12 samples for each stabilized soil mixture which allowed for multiple samples to be tested for each testing condition and curing duration.

The compression characteristics of the soils were determined using unconfined compression test procedures. The tests were conducted immediately after compaction (0-day tests), subsequent to 7 days of curing (7-day tests), and subsequent to 28 days of curing (28-day tests). The samples were cured in a moist chamber at a constant temperature and relative humidity. The velocities of the samples were determined for all testing conditions until the samples were subjected to an unconfined compression test. At a given curing age (0-day, 7-day, or 28-day) ultrasonic tests were conducted on all the available samples. After ultrasonic testing, three samples of each mixture were tested in an unconfined compression test. Using this procedure, more velocity data is available for the samples cured for a shorter duration. This is because after unconfined compression testing, samples are not 
available for further ultrasonic testing. Also, it was possible to monitor the variation of velocity with time and curing for the samples that were tested for compression characteristics subsequent to 7 days and 28 days of curing.

\section{Results and Discussion}

The variation of velocity with curing time for all the stabilized soil mixtures is presented in Figure 3. Average velocity of all the available samples tested at each testing condition is presented in the figure. Also, average velocity of only the samples that were tested for compression properties after 28 days of curing is presented in the figure. In this case, all of the velocity measurements were made on the same samples at the various testing times. The velocities obtained with the two approaches were very similar (Figure 3 ) and they can be used interchangeably to correlate velocities to engineering properties.

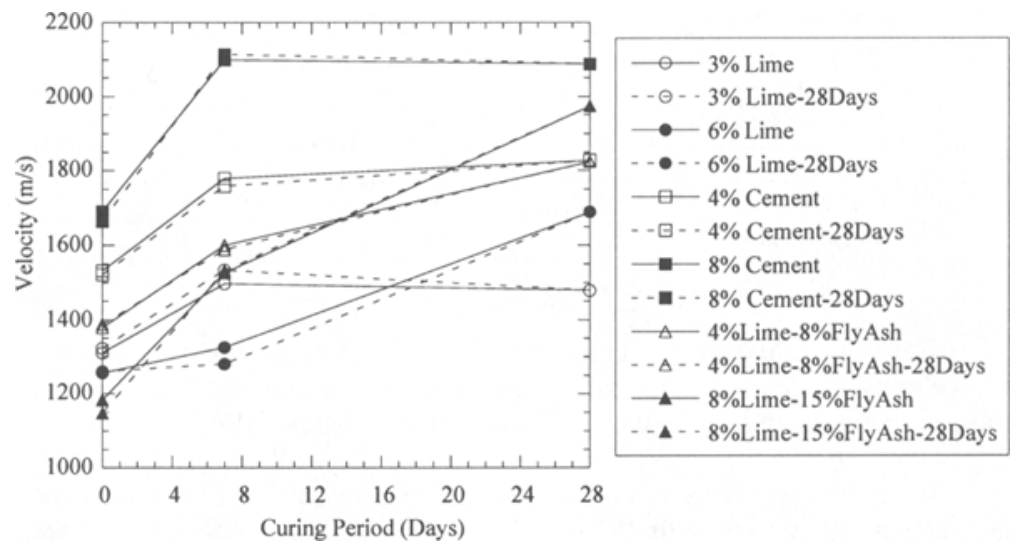

Figure 3. Variation of Velocity with Time

In general, velocities increased with curing time, and the increase from the day of compaction to 7 days was more pronounced than the increase from 7 days to 28 days. As the curing progresses, reactions occur between the soils and the stabilizing agents. These reactions generally result in increases in the stiffness of the soils. The velocity of wave propagation increases with increased stiffness of the soils. Also, it was observed that the velocities of soils stabilized with cement were higher than the velocities of the other mixtures. The velocities of the lime stabilized soils were in general lower than the velocities obtained for the other mixtures.

The variation of velocity with strength for all the samples is presented in Figure 4. Outer bounds of the observed trends are indicated on the plot. In general, velocity increased with strength of the soils. However, a significant amount of scatter was observed in the data. Variation of velocity with modulus was also 
investigated. Secant moduli at $1 \%$ strain, $2 \%$ strain, and $4 \%$ strain were determined for the samples using stress-strain relationships obtained from the compression tests. The variation of velocity with modulus is presented in Figure 5. It was observed that the velocity increased as the modulus of the samples increased. The relationships between velocity and various moduli for each stabilized mixture is presented in Figure 6. The quality of linear curve fits (as indicated by R-values) was better for individual mixtures (Figure 6) than for all mixtures plotted together (Figure 5). This is consistent with the individual mixtures having less inherent material variation.

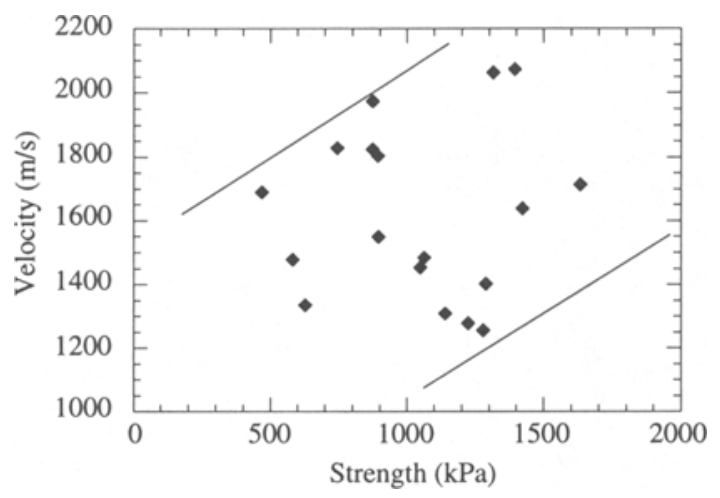

Figure 4. Variation of Velocity with Strength

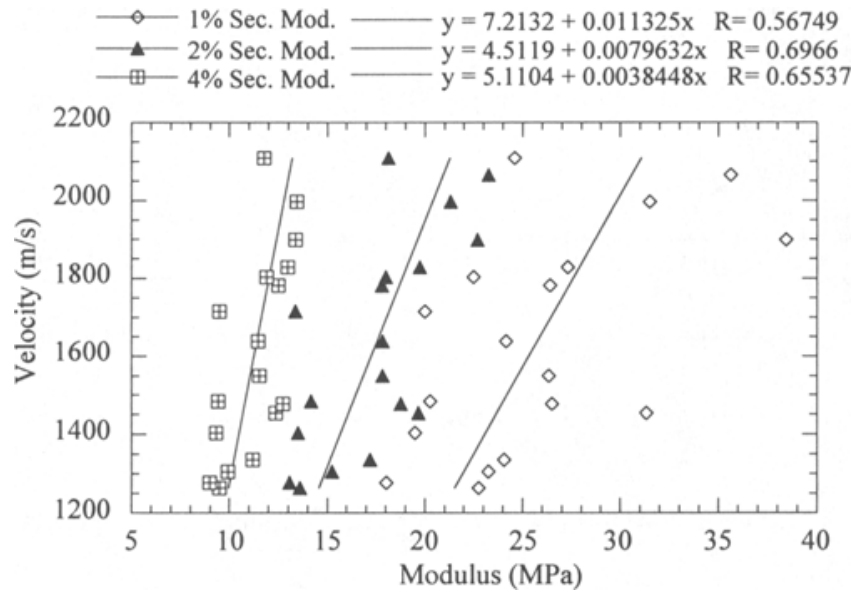

Figure 5. Variation of Velocity with Modulus 


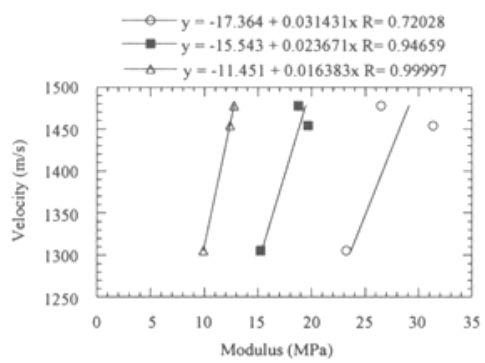

a) $3 \%$ Lime

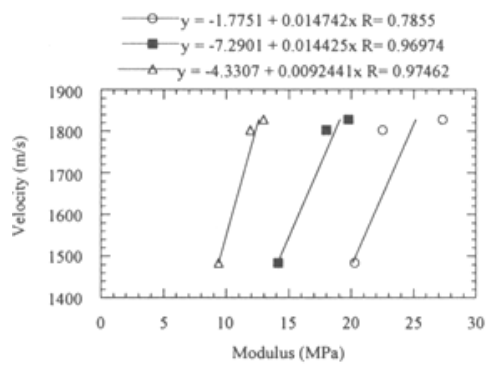

c) $4 \%$ Cement

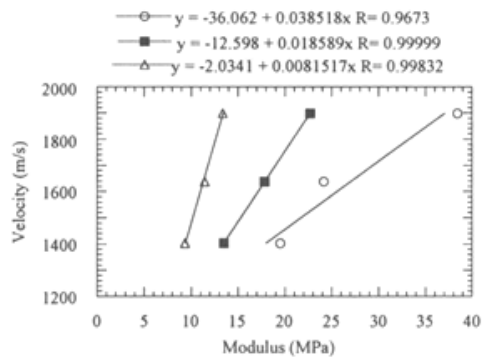

e) $4 \%$ Lime- $8 \%$ Fly Ash

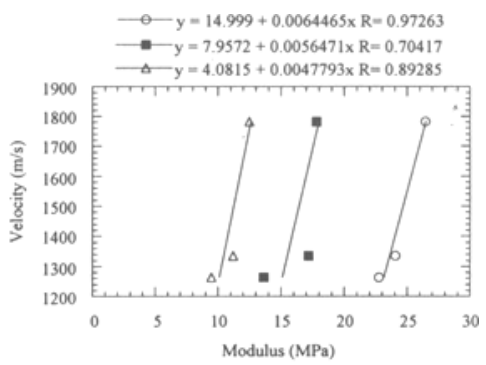

b) $6 \%$ Lime

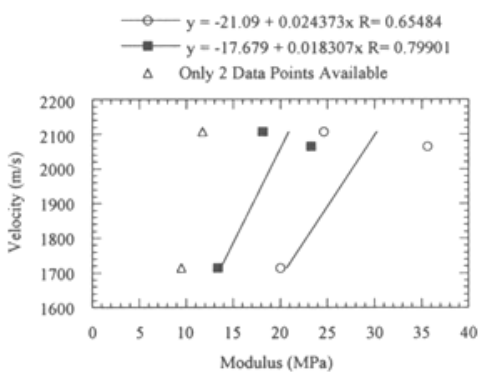

d) $8 \%$ Cement

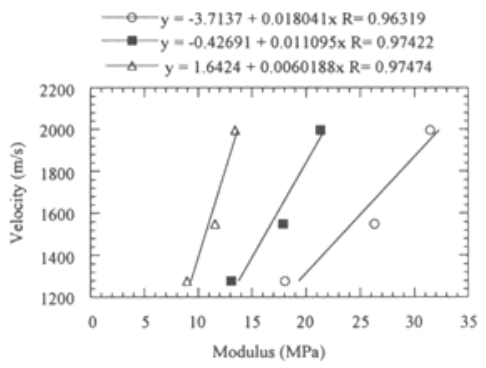

f) $8 \%$ Lime- $15 \%$ Fly Ash

The curve fits follow the order: $1 \%$ Secant Modulus, 2\% Secant Modulus, and 4\% Secant Modulus for all the plots.

Figure 6. Variation of Velocity with Modulus (Individual Mixtures) 
The trends observed in the velocity-modulus data were more pronounced than the trend observed in velocity-strength data. Wave propagation through soils occurs along the fastest path in the soil, which is directly correlated to the stiffness of the soil mass. The moduli represent the stiffness of the soil mass and hence are directly related to the velocity of wave propagation. Equations are available to determine the modulus for elastic materials using velocities of wave propagation. Strength of soils, on the other hand, represents an average property for the material that is affected by the composition of the material including voids, imperfections, and defects. Thus the strength of soils is not directly related to velocity of wave propagation although in general higher strengths can be expected to be associated with higher velocities. Similar observations have been reported for concrete (Naik and Malhotra 1991, Popovics and Popovics 1992).

The relationship of time vs. modulus and velocity is presented in Figure 7. The data for $4 \%$ secant modulus is presented in the figure as a typical example of the modulus-time relationship obtained for the soils. The changes in time for velocity and modulus were similar indicating that velocity measurements could be used to predict variation of modulus with curing time. The velocity was sensitive to the changes in modulus with curing time.
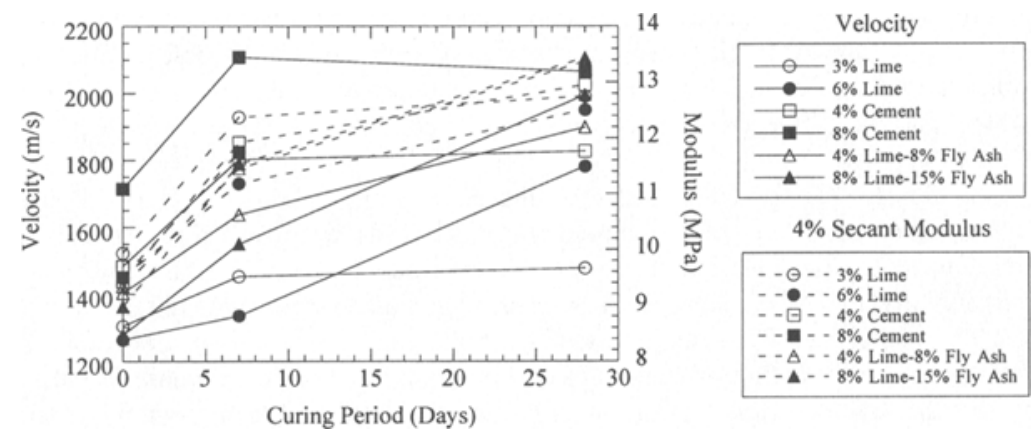

Figure 7. Variation of Velocity and Modulus with Time

In addition, the velocity increased with increasing dry and total density of the soils (Figure 8). The velocity of wave-propagation increases as the amount of solids in a soil mass increases and the amount of voids filled with air and water decreases. Similar observations were made for compacted clayey soils (Yesiller et al. 2000). 


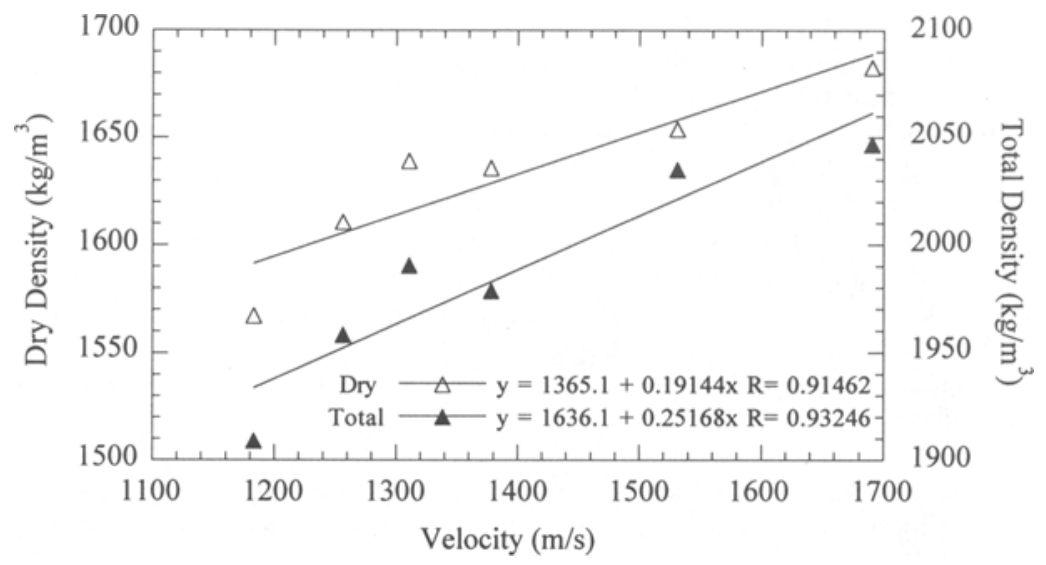

Figure 8. Variation of Velocity with Density

\section{Summary and Conclusions}

Tests were conducted to determine the feasibility of using ultrasonic testing to evaluate stabilized soils. P-wave velocities were determined on samples of a highplasticity clay soil stabilized with cement, lime, and lime - fly ash mixtures using commercially available electronic hardware. Compression characteristics of the samples were also determined at the day of compaction and subsequent to 7 days and 28 days of curing using unconfined compression tests.

Velocities of the stabilized soils increased with curing time with generally high velocities obtained for the cement stabilized soils and low velocities obtained for the lime stabilized soils. In general, the velocity of P-waves increased with increasing strength of the samples; however, significant scatter was observed in the data. The velocity of $\mathrm{P}$-waves increased with increasing modulus of the soils. The increase in velocity with modulus was more pronounced than the increase in velocity with strength. Wave transmission occurs along the fastest path in the soil, which is directly correlated to the stiffness of the soil mass or the modulus of the soil. The strength of a soil mass is affected by factors other than the stiffness of the soil including voids, imperfections, and defects and is not as closely correlated to velocity. In addition, changes in velocity and modulus in time was similar showing the sensitivity of the velocity measurements. Velocity of the soils also increased as the total and dry densities of the soils increased for the measurements conducted immediately after compaction of the samples. This is because wave transmission through solids is faster than through voids (filled with either air or water).

The ultrasonic test method is a fast and simple method that can be used to evaluate the characteristics of stabilized soils. The method also has the advantage of 
allowing for repeated measurements to be conducted on the same sample. The method can be adapted to testing of stabilized soils which could allow for reductions in the number of samples required for mixture design procedures and also can be adapted for field testing for determining characteristics and uniformity of the stabilized soils. Further research is needed to develop criteria to incorporate the ultrasonic test method into mixture design and field testing of stabilized soils.

\section{Acknowledgement}

Mr. Andrew Rener, who was partially funded by the Educational Foundation Scholarship of American Coal Ash Association, conducted the tests.

\section{References}

AASHTO, (1997). Standard specifications for transportation materials and methods of sampling and testing, part II tests, AASHTO, Washington, D.C.

ASTM, (1992). ASTM Standards on soil stabilization with admixtures, ASTM, West Conshohocken, PA.

Brown, R. W., Editor, (1996). Practical foundation engineering handbook, McGrawHill, New York, NY.

Inci, G., (2000). "Nondestructive evaluation of compacted clayey soils," Internal Report, Department of Civil and Environmental Engineering, Wayne State University, Detroit, Michigan.

McIntire, P., Editor, (1991). Nondestructive testing handbook, volume seven ultrasonic testing, American Society of Nondestructive Testing, Columbus, $\mathrm{OH}$.

Naik, T. R., and Malhotra, V. M., (1991). "The ultrasonic pulse velocity," Handbook on nondestructive testing of concrete, V. M. Malhotra and N. J. Carino, Eds., CRC Press, 169-187.

Popovics, S., and Popovics, J. S., (1992). "A critique of the ultrasonic pulse velocity method for testing concrete," Nondestructive testing of concrete elements and structures, F. Ansari and S. Sture, Eds., ASCE, Reston, VA.

Udegbunam, O., (2000), "Ultrasonic assessment of service life of concrete structures subject to reinforcing steel corrosion," Ph.D. Thesis, Department of Civil and Environmental Engineering, Wayne State University, Detroit, Michigan.

Yesiller, N., Inci, G., and Miller, C. J., (2000), "Ultrasonic testing for compacted clayey soils," Advances in Unsaturated Geotechnics, ASCE GSP 99, C. D. Shackelford, S. L. Houston, and N.-Y. Chang, Eds., ASCE, 54-68. 